\title{
Les universités et les gestionnaires autochtones : concevoir un partenariat de formation pour soutenir l'autonomie gouvernementale
}

Jean-François Savard

\section{(2) OpenEdition Journals}

Édition électronique

URL : http://journals.openedition.org/ripes/599

DOI : 10.4000/ripes.599

ISSN : 2076-8427

Éditeur

Association internationale de pédagogie universitaire

Référence électronique

Jean-François Savard, "Les universités et les gestionnaires autochtones : concevoir un partenariat de formation pour soutenir l'autonomie gouvernementale », Revue internationale de pédagogie de

l'enseignement supérieur [En ligne], 28(1) | 2012, mis en ligne le 20 avril 2012, consulté le 07 septembre 2020. URL : http://journals.openedition.org/ripes/599 ; DOI : https://doi.org/10.4000/ripes.599

Ce document a été généré automatiquement le 7 septembre 2020

Article L.111-1 du Code de la propriété intellectuelle. 


\title{
Les universités et les gestionnaires autochtones : concevoir un partenariat de formation pour soutenir l'autonomie gouvernementale
}

\author{
Jean-François Savard
}

\section{Introduction}

1 Depuis les années '70, le Canada a connu un fort mouvement de revendications en faveur d'une plus grande autonomie gouvernementale autochtone. Cette autonomie repose sur trois piliers que sont l'autorité légale, le financement et l'administration publique. C'est pourquoi, dans les années ' 80 et ' 90 , le Canada s'est doté d'outils légaux et politiques qui permettent de reconnaître des autorités légales pour la mise en œuvre de l'autonomie gouvernementale autochtone et des programmes de transfert de fonds qui visent à financer cette autonomie. Cependant, bien que les autochtones puissent jouir d'autorités légales et de fonds pour soutenir leur autonomie gouvernementale, ils ont un besoin criant de programmes universitaires pour former leurs gestionnaires et mettre sur pied une administration publique compétente.

2 Cet article rend compte de deux projets de partenariat entre une université québécoise, l'École nationale d'administration publique (ENAP), et deux organisations autochtones, le Conseil Cri ${ }^{1}$ de la santé et des services sociaux de la Baie-James (CCSSSBJ) et le Conseil en éducation des Premières nations (CEPN). L'ENAP a pour mission d'offrir des programmes de maitrise en administration publique adaptés aux réalités sociales et culturelles des autochtones. Ces programmes ont été conçus non seulement à la demande de ces organisations, mais en partenariat direct avec elles. L'objectif est de former, à terme, des professionnels de l'administration publique qui pourront 
contribuer positivement à la gestion de l'autonomie gouvernementale de leurs communautés.

3 Les programmes présentés dans cet article sont soit en cours de réalisation, soit en phase de démarrage. Le but de ce texte n'est donc pas d'exposer des résultats finaux, mais bien de démontrer comment une démarche particulière de partenariat entre université et organisations autochtones a pu donner naissance à des programmes adaptés aux réalités et aux besoins sociaux et culturels de ces derniers. Pour ce faire, nous exposerons, d'abord, la méthodologie de notre analyse des pratiques, puis nous exposerons les éléments qui, à la suite de nos recherches, ressortent comme étant essentiels dans la conception d'un dispositif de formation et d'un modèle pédagogique adapté à des clientèles autochtones. Nous présenterons, ensuite, la démarche de partenariat entre l'ENAP et le CSSSCBJ, ainsi que celui conclu avec le CEPN. Nous discuterons, finalement, les modèles pédagogiques développés par l'ENAP en partenariat avec les organisations autochtones et l'expression des avis des autochtones à l'égard de ces modèles.

\section{Une approche basée sur l'observation participante}

4 Notre analyse de pratiques est fondée sur trois méthodes à caractère ethnographique : 1) l'observation participante ; 2) la consultation et 3) l'étude de cas (Gagnon, 2008 ; Gauthier, 2009 ; Karsenti \& Savoie-Zajc, 2004 ; Thiétart, 2003). De fait, l'analyse s'appuie principalement sur les observations que nous avons faites des démarches d'élaboration en partenariat de deux programmes qui visent à habiliter, d'une part, des gestionnaires du Conseil Cri de la santé et des services sociaux de la Baie-James (CSSSCBJ) et, d'autre part, des gestionnaires de différentes communautés des Premières nations membres du Conseil en éducation des Premières nations (CEPN). À travers ces démarches, qui ont démarré respectivement en 2006 et en 2009, nous avons pu observer les problèmes et les lacunes systémiques qui rendent difficile la mise en place de programmes de formation pour des gestionnaires autochtones. Notre analyse tient aussi compte d'une consultation menée auprès des étudiantes et étudiants membres du CEPN à la suite des deux premiers cours qu'ils ont suivis dans le cadre de leur programme. Cette consultation visait à connaître le niveau de satisfaction vis à vis du modèle pédagogique proposé.

5 L'observation participante et la consultation auprès des étudiants nous ont permis d'étudier la démarche menant à l'élaboration de deux programmes de formation pour des gestionnaires autochtones inscrits à l'École nationale d'administration publique (ENAP) : la maitrise en administration publique pour les gestionnaires du CCSSSBJ et un programme court de formation continue en management public pour les gestionnaires du CEPN. Dans un cas comme dans l'autre, nous allons voir comment l'ENAP a tenu compte d'éléments spécifiques afin de créer un modèle pédagogique qui respecte les réalités particulières de la clientèle autochtone. À cet égard, il est pertinent de noter que le programme pour les gestionnaires du CCSSSBJ démarre en 2012, alors que celui pour les gestionnaires membres du CEPN a débuté à l'automne 2010.

6 Notre propos est celui d'un praticien en prise avec cette réalité particulière où une université dialogue avec des peuples autochtones. C'est en coordinateur du terrain que nous nous posons. Nous allons essentiellement rendre compte de notre expérience et de nos observations. Nous nous distancions en cela des contributions plus conceptuelles 
et théoriques qui composent ce numéro thématique, ce qui explique le faible référencement de l'article et l'approche résolument descriptive.

\section{Le contexte d'élaboration du partenariat}

7 Les universités canadiennes ont mis en œuvre plusieurs programmes de formation à l'intention des communautés autochtones, mais très peu d'entre eux visent le développement de compétences en gestion (Savard, Moreau, \& Jacob, 2010). Sur la base de nos expériences nous avons pu identifier quatre éléments qui favorisent la mise en place de programmes universitaires conçus pour les autochtones. Ces quatre éléments sont interdépendants et fondent le modèle pédagogique de référence.

Le premier élément est la mise en place d'une architecture particulière pour les programmes et dispositifs de formation conçus spécifiquement pour la clientèle autochtone. Pour ce qui concerne les programmes, on retrouve généralement deux cas de figure dans les universités canadiennes. Certaines universités offrent des programmes préparatoires dont la réussite est requise pour être ensuite admis dans un programme régulier. Le programme préparatoire sert de mise à niveau pour les participants par l'acquisition des compétences nécessaires aux études universitaires et leur évaluation. Le programme préparatoire prévoit habituellement la possibilité pour les participants de passer directement au programme universitaire, sans avoir à présenter une nouvelle demande d'admission. La stratégie de passerelle permet parfois de reconnaître les cours du programme préparatoire comme faisant partie intégrante d'un programme régulier. Il s'agit d'une mesure d'encouragement à l'intention des participants.

9 Ensuite, certaines universités adoptent une stratégie dite d'empilement (building block en anglais) qui permet d'obtenir, à petits pas, un baccalauréat canadien $\left(1^{\mathrm{er}}\right.$ cycle universitaire). La logique de l'empilement permet d'éviter le décrochage des études et de favoriser la réussite. En effet, l'expérience montre que cette approche permet aux autochtones d'obtenir une reconnaissance universitaire, étape par étape, en évitant le découragement au cours du programme perçu comme interminable, tout en les maintenant dans des programmes de formation de plus en plus spécialisés et exigeants. L'approche est d'autant plus indiquée lorsque l'on offre une formation à des gestionnaires en poste. Comme ces derniers poursuivent des études à temps partiel, un programme de formation universitaire traditionnel parait trop long à compléter, ce qui entraîne un sentiment de découragement.

10 Le deuxième élément est le développement de services personnalités aux étudiants autochtones. Il s'agit de services qui permettent à la communauté étudiante autochtone de se retrouver (café étudiant, association étudiante, centre culturel, etc.) hors des dispositifs de formation, mais aussi de services de soutien pédagogique qui font appel aux valeurs autochtones telles que le respect des aînés (programmes de mentorat, rencontres avec des aînés, etc.). Ces services hors cadre universitaire sont très importants, particulièrement pour les gestionnaires en poste. En effet, ces derniers peuvent se sentir souvent isolés et éprouver des difficultés à concilier le travail et les études. Le soutien peut prendre la forme de personnes ressources dans la communauté, d'une ligne d'appel, de la mise en place d'un réseau spécifique, etc. L'important est de garder un lien constant entre l'étudiant et l'université pour soutenir la motivation à compléter le programme. 
11 Le troisième élément est l'adoption d'une approche qui lie des dispositifs de formation continue aux programmes universitaires. Le principe est assez simple. Il s'agit de reconnaître les compétences et connaissances acquises dans un dispositif de formation continue à l'intérieur d'un programme universitaire régulier. L'avantage d'une telle stratégie est de permettre plus aisément la formation des gestionnaires en poste, de flexibiliser leurs parcours de formation et de leur offrir un accompagnement individualisé.

Enfin, le quatrième élément touche à la modularisation des cours. En effet, comme un grand nombre de communautés autochtones habitent dans des régions éloignées ou isolées, la façon de dispenser les cours à l'intérieur du programme doit, elle aussi, être adaptée. La flexibilité des cours et des dispositifs formatifs de même que l'utilisation des technologies d'information et de communication invitent à franchir les obstacles que représentent l'éloignement et l'étalement.

13 La figure 1 illustre l'articulation des quatre éléments dans la conception et le développement d'un programme.

Figure 1 : Les quatre éléments d'un programme d'enseignement ou de formation continue

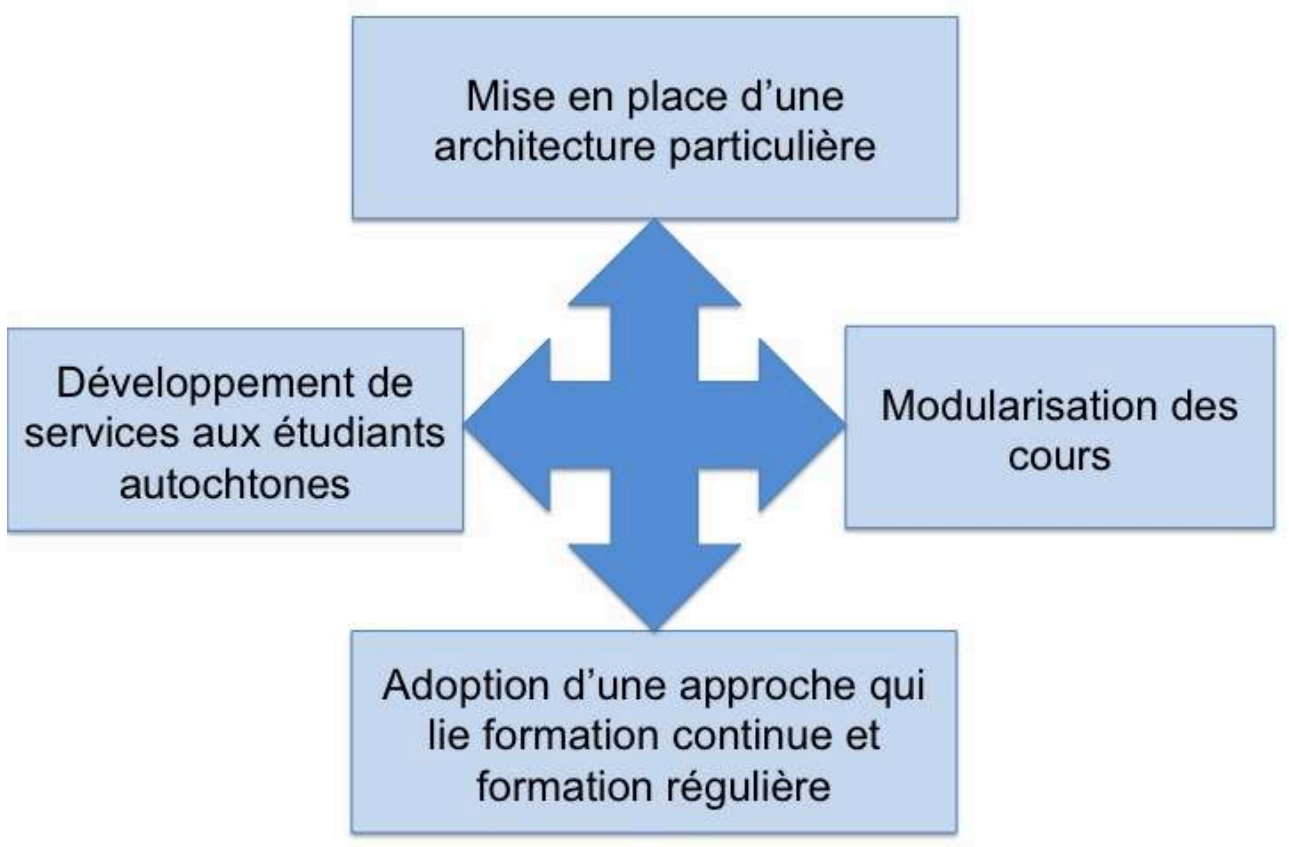

\section{La démarche de partenariat}

14 L'ENAP est une université spécialisée vouée au développement de l'administration publique à la fois comme champ de connaissance et comme champ de pratique. Elle offre une formation universitaire avec des programmes de maîtrise et de doctorat et elle représente, ce faisant, un pôle de recherche important en administration publique. En 2006, l'ENAP a choisi de s'engager plus activement dans l'appui à la gouvernance en milieu autochtone en mettant en place des programmes de formation pour des 
gestionnaires autochtones. Ces programmes visent non seulement à développer les connaissances de la gestion publique pour des gestionnaires déjà en fonction, mais également à ce qu'ils obtiennent un diplôme de deuxième cycle universitaire.

\subsection{Le premier partenariat : un projet sur mesure}

Le premier projet a démarré en 2006. Suite à une demande formulée par les autorités du Conseil Cri de la santé et des services sociaux de la Baie-James (CCSSSBJ), l'ENAP est entrée en matière pour développer un programme universitaire " sur mesure " pour les gestionnaires de cette organisation. Le programme élaboré a été négocié en deux temps. Dans un premier temps, le mandat confié à l'ENAP portait sur la formation à la gestion des personnes en fonction afin de leur faciliter l'accès aux études universitaires, tout en les soutenant dans l'exercice de leurs fonctions. Le but, ici, était de leur proposer un programme préparatoire aux études universitaires.

Dans un deuxième temps, l'ENAP a été invitée par le CCSSSBJ à élaborer une entente pour offrir un programme de maîtrise en administration publique à ses gestionnaires. Si au départ la capacité de ces étudiants à entreprendre un programme universitaire inquiétait les responsables de l'ENAP et du CCSSSBJ, à la suite du programme préparatoire, c'était surtout la persévérance des étudiants autochtones qui retenait l'attention. L'ENAP a donc proposé au Conseil d'adopter une stratégie d'empilement (building block). L'ENAP propose trois programmes distincts de formation au deuxième cycle universitaire : le programme court (15 crédits), le diplôme d'études supérieures spécialisées (DESS de 30 crédits) et la maîtrise ( 45 crédits). L'étudiant qui veut passer d'un programme à l'autre doit déposer, à chaque fois, une nouvelle demande d'admission, puisque ces programmes sont distincts. La figure 2 illustre cette progression possible à l'intérieur des programmes. 


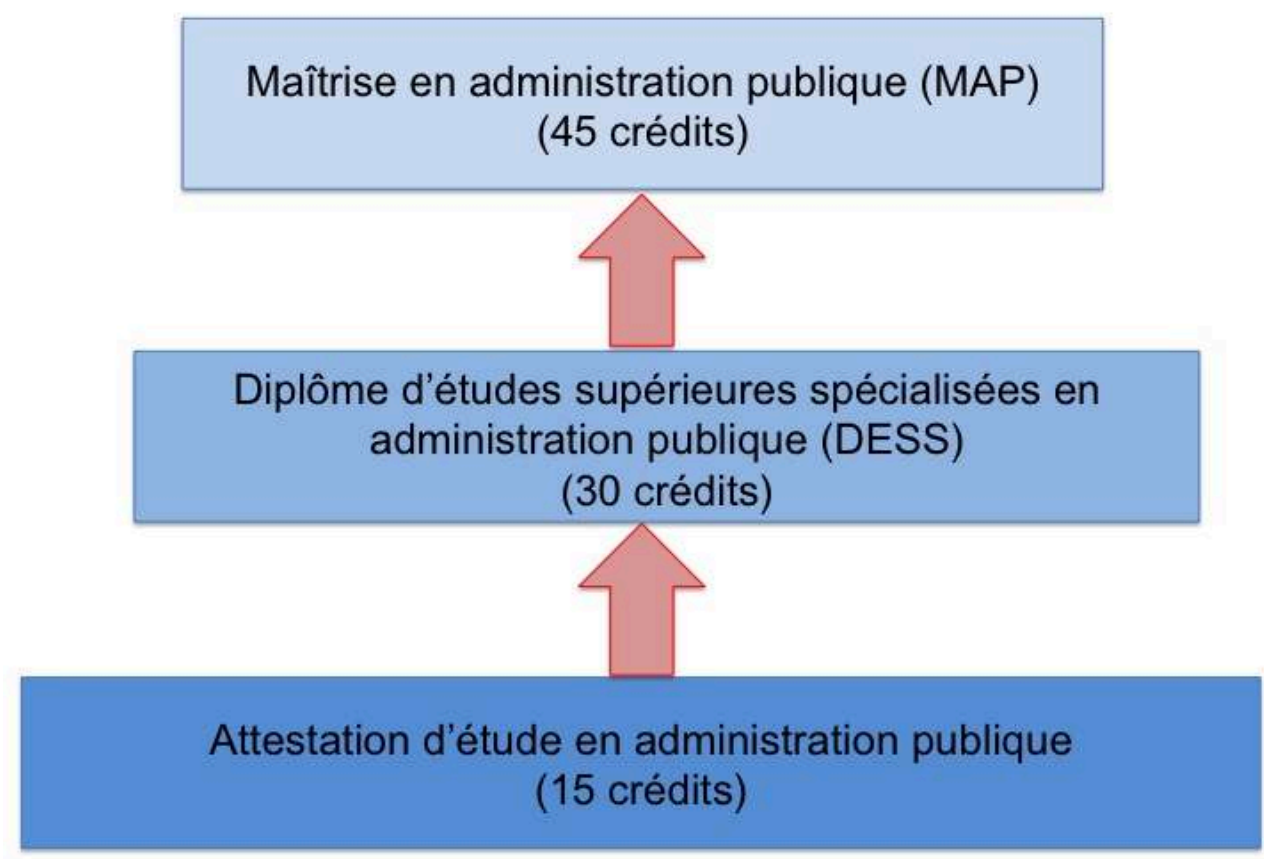

Dans le cadre de l'entente avec le CCSSSBJ, l'ENAP a proposé un parcours continu au cours duquel les étudiants poursuivent leur formation par étape de 15 crédits selon le système de crédits en vigueur dans les universités québécoises. À chaque étape, l'étudiant peut décider de conclure sa formation et obtenir la reconnaissance de l'ENAP pour les étapes du parcours validées. Ainsi, un étudiant qui complètera 15 crédits obtiendra une attestation d'études, celui qui complètera 30 crédits un diplôme d'études supérieures spécialisées en administration publique et celui qui terminera les 45 crédits obtiendra une maitrise en administration publique. En découpant ainsi le cursus de formation en trois étapes avec reconnaissance possible des études achevées à chacune des étapes, l'ENAP et le CCSSSBJ s'efforcent de diminuer le découragement et de favoriser la persévérance aux études.

L'accord prévoit également que l'ENAP et le CCSSSBJ mettent à la disposition des étudiants des conseillers. Ces derniers auront pour fonction de les soutenir dans les périodes difficiles et de les accompagner pour surmonter les éventuels obstacles qu'ils pourraient rencontrer. Cet accompagnement pédagogique constitue une autre stratégie favorisant la persévérance aux études.

Enfin, notons que les cours eux-mêmes sont structurés de façon particulière. L'ENAP offre généralement ses cours sur une période de 15 semaines, à raison d'un cours de trois heures par semaine et les professeurs tiennent différentes activités d'évaluation au cours de ces 15 semaines. Dans le cadre de l'entente avec le CCSSSBJ, l'ENAP s'est engagé à dispenser les cours de manière intensive sur une période de deux semaines. Les professeurs seront invités à faire parvenir aux étudiants, quelques semaines auparavant, tout le matériel de lecture et d'exercice, de tenir une évaluation au cours de ces deux semaines, puis une dernière évaluation à remettre quelques semaines après le cours intensif. 
20 L'entente avec le CCSSSBJ a été conclue fin 2011 et le programme débute en 2012. Il est donc trop tôt pour dire si ce dispositif de formation répond entièrement aux attentes et besoins identifiés. Or, le principe d'un programme de formation échelonné semble tenir compte des conditions nécessaires et connues pour soutenir la réussite des étudiantes autochtones.

\subsection{Un deuxième partenariat pour le Conseil en éducation des Premières nations}

21 Le deuxième projet développé pour la gouvernance autochtone a démarré en 2009, lorsque le Conseil en éducation des Premières nations (CEPN) a demandé à l'ENAP d'élaborer un programme de formation universitaire pour les gestionnaires des conseils de bande et des conseils tribaux. Suite à l'analyse des besoins de ces gestionnaires et compte tenu des différentes options que l'ENAP pouvait offrir, le CEPN a opté pour un programme court plutôt qu'un programme de maîtrise.

22 Le dispositif de formation développé dans le cadre de cette entente pour un programme court est assez simple. Le programme comprend cinq cours totalisant 15 crédits chacun qui représentent la première étape du programme du CCSSSBJ. Les cours répondent spécifiquement aux besoins identifiés par les gestionnaires autochtones. Lorsque les étudiants auront complété les 15 crédits prévus au programme, ils obtiendront une attestation d'études et pourront alors déposer une demande d'admission à un programme de DESS ou de maîtrise.

23 Tout comme dans le cas du projet mené avec le CCSSSBJ, le CEPN a mis en place, avec la collaboration de l'ENAP, une structure de soutien pour aider les étudiants à persévérer dans leurs études. Plus précisément, le CEPN a composé une équipe de soutien informatique pour aider les étudiants qui auraient des difficultés dans le domaine et désignée une conseillère qui veille au bon déroulement du programme et qui est à disposition des étudiants si ces derniers devaient rencontrer des difficultés particulières. De son côté, l'ENAP met à la disposition des étudiants inscrits dans ce programme un professeur au titre de conseiller ou de mentor avec comme fonction spécifique de soutenir les étudiants qui éprouveraient des difficultés lors de la production de leurs travaux ou des problèmes de compréhension dans leurs lectures.

Ce qui distingue le programme du CEPN du programme du CCSSSBJ c'est la modularisation des cours et l'organisation des enseignements. Pour les cours aux gestionnaires membres du CEPN, l'ENAP devait composer avec une clientèle dispersée sur tout le territoire québécois, donc se trouvant dans des régions éloignées, voire souvent isolées. Le CEPN ne désirait pas de cours intensifs sur une période de deux semaines. L'ENAP a ainsi dû faire preuve de créativité pour organiser les enseignements. Pour les neuf premières heures de cours, tous les étudiants doivent être présents au siège social du CEPN qui se trouve à Wendake ${ }^{2}$ dans la région de la ville de Québec. Les trente-six heures suivantes sont offertes en visioconférence, permettant ainsi aux étudiants de suivre la formation à partir de leur communauté ou de leur foyer. Le système de visioconférence du CEPN fonctionne à la fois sur des appareils fixes et avec des ordinateurs personnels et vise à créer une classe en mode virtuel. Les professeurs peuvent projeter des diaporamas ou encore utiliser un tableau blanc interactif. 
25 Le programme court pour le CEPN a débuté à l'automne 2010. Il est trop tôt pour en faire une évaluation complète mais, comme nous le verrons dans la section suivante, nous pouvons d'ores et déjà fournir quelques éléments intéressants par rapport à cette expérience.

\section{Un modèle pédagogique adapté aux spécificités autochtones}

\subsection{L'architecture des programmes mis en place}

Il se dégage de la démarche de partenariat mis en place entre l'ENAP et les organismes autochtones, un modèle pédagogique qui intègre les éléments essentiels au respect des réalités et besoins propres aux gestionnaires autochtones. Dans les deux ententes étudiées, l'ENAP adopte une architecture de programme particulière pour répondre aux besoins exprimés par les organismes autochtones. Dans le cas du CCSSSBJ, elle a mis en place un programme préparatoire pour permettre aux gestionnaires Cris d'acquérir et de peaufiner les habiletés nécessaires à la réussite d'une formation universitaire. Il faut souligner que ce programme préparatoire répond non seulement à la nécessité d'une architecture particulière, mais vise également à inclure des modules de formation continue à l'intérieur d'un programme de formation universitaire régulière. En effet, comme l'ENAP ne prévoit pas de programme préparatoire dans sa formation régulière, ce programme a été offert au titre de formation continue, Or, l'entente prévoit que les étudiants qui réussissent le programme préparatoire peuvent se voir reconnaitre ces crédits dans leur formation universitaire régulière. C'est à travers cette stratégie de passerelle que l'on peut lier la formation continue à la formation universitaire.

L'entente avec le CCSSSBJ prévoit aussi que la formation universitaire sera offerte selon une logique d'empilement (building block) pour éviter le découragement des étudiants. Dans l'entente avec le CEPN, la particularité de l'architecture n'est pas aussi importante que dans l'entente avec l'organisme Cri, simplement parce qu'il s'agit d'un programme court qui ne compte que 15 crédits. Néanmoins, l'entente prévoit une architecture particulière, puisque contrairement à l'usage habituel de l'ENAP où chaque étudiant est responsable de son choix de cours, dans l'entente avec le CEPN, c'est l'organisme qui a sélectionné les cinq cours que devront suivre les gestionnaires et l'ordre dans lequel ces cours sont offerts, en fonction des besoins des communautés autochtones.

\subsection{Les dispositifs d'accompagnement de la démarche}

En ce qui a trait aux services aux étudiants, les deux ententes prévoient des prestations particulières pour favoriser la réussite des étudiants autochtones. L'entente avec le CCSSSBJ propose d'offrir aux étudiants des conseillers Cris pour les soutenir dans les moments plus ardus (par exemple, quand il faut concilier travail, famille et études) et des conseillers pédagogiques de l'ENAP pour les aider à développer leurs habiletés d'études (écriture scientifique, lectures, etc.). L'entente avec le CEPN offre aux étudiants l'appui d'une équipe informatique (pour répondre aux questions relatives à l'utilisation des ordinateurs et des logiciels), d'une conseillère autochtone qui appuie 
les étudiants de la même façon que le conseiller Cri et un conseiller pédagogique de l'ENAP qui a les mêmes fonctions que dans l'entente avec le conseil Cri.

Enfin, notons que dans le cadre des deux ententes, l'ENAP s'est engagé à offrir une modularisation de l'enseignement pour répondre aux particularités géographiques des populations autochtones. Les cours qui sont offerts aux gestionnaires du CCSSSBJ se déroulent sur des périodes intensives de deux semaines avec des lectures préalables au cours et des travaux à remettre après la fin du cours. Les cours offerts aux gestionnaires du CEPN se déroulent en présence pour les neuf premières heures, puis en visioconférence. On voit, ici, la volonté de l'ENAP de mettre en place un modèle pédagogique particulier qui s'appuie sur le principe de la flexibilité.

Nous avons mentionné, dans la section précédente, que le programme crédité pour les gestionnaires du CCSSSBJ est sur le point de démarrer, alors que celui pour les gestionnaires du CEPN vient à peine de débuter. Difficile dans ces conditions de tirer un bilan complet des expériences. Cependant, le programme préparatoire pour les gestionnaires du CCSSSBJ a déjà été complété et les gestionnaires du CEPN ont déjà terminé quatre cours de leur programme. Il est donc possible d'en dresser un bilan provisoire.

\subsection{Le bilan du programme préparatoire à destination des gestionnaires du CCSSSBJ}

31 Au total 31 personnes ont suivi le programme préparatoire pour les gestionnaires du CCSSSBJ. Seules deux personnes n'ont pas pu le compléter. Les activités se sont tenues à Montréal, à Gatineau et à Val-d'Or (des villes réparties d'est en ouest sur le territoire québécois) à la demande des responsables du Conseil, ce qui permettait, d'abord, de réunir en un seul lieu tous les étudiants et passer outre l'obstacle que représente l'étalement géographique, puis d'obliger les participants à se concentrer sur leur formation en les libérant de leurs tâches quotidienne de gestion, puisqu'ils devaient se déplacer pour suivre le programme. De plus, l'ENAP et le CCSSSBJ se sont entendus pour mettre sept personnes ressources à la disposition des étudiants, qui sont intervenues tout au long du programme pour assurer la poursuite des études et la réussite des participants.

32 À la fin du programme, les participants ont tous été invités à répondre à un questionnaire qui fut administré en salle de cours, afin de s'assurer que tous les étudiants y participent. Le questionnaire comptait trente énoncés touchant divers aspects de la formation pour lesquels les participants devaient indiquer leur degré d'accord sur une échelle de 1 à 4,1 correspondant à un total accord et 4 à un total désaccord. Un espace libre a été prévu pour que les participants puissent faire des commentaires libres. Le taux de satisfaction a été établi en calculant le pourcentage de réponses positives (score 1 et 2) pour chaque item. Le taux de satisfaction globale s'élève à $86 \%$, soit la moyenne des taux obtenus pour les trente énoncés du questionnaire.

\subsection{Premier bilan du programme court du CEPN}

33 Le programme court du CEPN comptait au départ 23 personnes, dont 18 qui poursuivent toujours le programme. Une première consultation a été menée auprès de 
ces étudiants pour connaître leurs impressions par rapport à la formation. Cette consultation a pris la forme d'un questionnaire administré de façon électronique à tous les étudiants inscrits dans le programme. Le questionnaire comptait trois questions ouvertes auxquelles les étudiants étaient invités à répondre. Des dix-huit étudiants ayant reçu le questionnaire par courriel, seule la moitié a répondu. Avec seulement neuf réponses en retour, il faut nuancer les résultats et les considérer comme des indications de préférence générales. Les impressions des étudiants portent seulement sur la modularisation de l'enseignement et sur l'utilisation de la technologie. Pour les autres composantes du programme, il est encore trop tôt pour les évaluer. Les étudiants répondants apprécient cette première expérience. Ils ont souligné l'importance de pouvoir suivre leur formation en deux temps, à savoir en présence et à distance. À cet égard, un étudiant soulignait que «la possibilité de suivre des cours universitaires à distance en direct [...] demeure pour moi un grand avantage, surtout s'il faut concilier travail à temps plein, famille et études ».

Les étudiants ont également relevé plusieurs points positifs à cette modularisation de l'enseignement. D'abord, la visioconférence permet de pallier les longues distances (des milliers de kilomètres pour certains) et de réduire ainsi les voyages. Certains parlent même d'une démocratisation de la formation universitaire, puisqu'elle ne se trouve plus limitée aux seules personnes qui habitent les grands centres urbains, mais qu'elle peut se déployer dans les régions les plus reculées du pays. D'autres avantages ont été soulignés, comme la conciliation travail-famille-études, l'utilisation efficiente des ressources (les cours en visioconférence qui permettent d'éviter de prendre congé, de réduire les dépenses liées aux voyages et d'assurer une utilisation plus efficace du temps des gestionnaires) et l'apprentissage des communications numériques (en effet, certains étudiants ont vu dans l'utilisation de la visioconférence une possibilité d'acquérir une habileté nouvelle qui pourrait leur être utile dans leurs communications avec d'autres communautés).

Cela dit, l'utilisation de la visioconférence n'a pas que des avantages. Les étudiants ont relevé certains points négatifs, comme le manque de contacts humains et la difficulté à interagir en groupe. La classe virtuelle connaît donc des limites et ne peut rendre intégralement l'expérience d'une classe en présentiel. À cet égard, des étudiants ont souligné que les neuf premières heures d'enseignement en présence étaient nécessaires et très utiles afin de créer un esprit de groupe, ce qui rend, par la suite, l'usage de la visioconférence plus efficace. Enfin, plusieurs étudiants ont souligné qu'ils avaient plus de difficulté à se concentrer lorsque les cours étaient en visioconférence, soit parce que les éléments de distraction sont plus nombreux qu'en classe, soit parce que le fait de se concentrer sur un écran exige plus d'énergie et induit une fatigue plus rapide qu'en salle de classe.

Si l'enseignement présente certains éléments négatifs, les étudiants soulignent les aspects positifs et concluent que l'expérience est satisfaisante. Plusieurs d'entre eux ont relevé que l'enseignement en visioconférence exige une pédagogie adaptée pour être porteuse. Les exercices en groupe notamment sont difficiles à réaliser en visioconférence. Les professeurs doivent éviter les longues envolées oratoires, puisque les étudiants perdent plus facilement et plus rapidement leur concentration. Il semble préférable d'adopter un ton particulièrement stimulant et de favoriser autant que possible les interactions entre les participants de la visioconférence. Les étudiants ont tendance à fermer leurs microphones pour éviter que du bruit ne vienne couper le son 
d'une personne qui parle. Or, cette façon de faire réduit considérablement le sentiment d'apprendre collégialement et d'appartenir à un groupe, entraîne plus facilement la perte de concentration et limite la portée de la dynamique collective d'apprentissage.

\section{Conclusion}

37 Cet article avait pour but de rendre compte d'une démarche particulière de partenariat visant à former des gestionnaires autochtones, afin de favoriser la gestion de leur autonomie gouvernementale. Nous avons passé en revue quelques expériences canadiennes de programmes universitaires élaborés pour répondre aux besoins des autochtones et en avons tiré quatre éléments essentiels qui doivent s'articuler au sein d'un modèle pédagogique spécifique aux réalités et aux besoins autochtones.

Ce bilan suggère que la démarche de partenariat entre l'ENAP et les organisations autochtones conduit à l'adoption d'un modèle pédagogique qui, jusqu'à présent, répond pleinement aux réalités et besoins spécifiques des autochtones favorisant leur réussite.

Cette analyse voulait faire état d'une pratique innovante à l'ENAP qui, à terme, aura des effets bénéfiques sur le plan social et politique pour les autochtones en améliorant la gestion de leur autonomie gouvernementale par la formation de leurs gestionnaires. Cette pratique prend son sens à travers une démarche particulière de partenariat où l'ENAP est invité par les organisations autochtones elles-mêmes à négocier l'élaboration et la mise en œuvre de programmes de formation universitaire. Ces deux expériences sont encore en cours de réalisation et il est trop tôt pour en évaluer l'efficacité et surtout généraliser les conclusions. Toutefois, les résultats préliminaires de nos observations et de nos consultations laissent à penser que ce type de partenariat et ce type de modèle pédagogique constituent une voie d'avenir pour la formation universitaire des gestionnaires autochtones. Notre prochaine étape sera d'attendre la fin de ces deux programmes pour en faire une évaluation complète et exhaustive.

\section{BIBLIOGRAPHIE}

Gagnon, Y.-C. (2008). L'étude de cas comme méthode de recherche. Québec : Presses de l'Université du Québec.

Gauthier, B. (2009). Recherche sociale : de la problématique à la collecte de données. Québec : Presses de l'Université du Québec.

Karsenti, T., \& Savoie-Zajc, L. (2004). La recherche en éducation : étapes et approches. Sherbrooke : Éditions du CRP.

Savard, J.F., Moreau, M., \& Jacob, M. (2010). Sortir des sentiers battus : repenser le rôle des universités dans le soutien à l'autonomie gouvernementale autochtone Dans J. White \& J. Peters (dir.), Aboriginal Policy Research (p. 29-53). Toronto : Thompson Educational Publishers.

Thiétart, R. A. (2003). Méthodes de recherche en management. Paris : Dunod. 


\section{NOTES}

1. Les Cris sont une nation autochtone de l'Amérique du Nord. Les Cris du Québec sont réunis au sein du Grand Conseil des Cris et le peuple se répartit en cinq communautés le long du littoral est de la Baie James.

2. Le CEPN dispose de salles de classe intégrant les plus récentes technologies d'information et de communication adaptées à l'enseignement universitaire. Il s'agit donc d'un endroit indiqué pour offrir une formation universitaire.

\section{RÉSUMÉS}

Aux prises avec des besoins particuliers de formation de leurs gestionnaires pour soutenir la gestion de leur autonomie gouvernementale, les communautés autochtones se tournent aujourd'hui vers les universités pour conclure des partenariats de formation. Cet article rend compte de deux partenariats conclus, au Québec, entre l'École nationale d'administration publique (ENAP) et le Conseil du peuple Cri de la Baie-James, d'une part, et le Conseil en éducation des Premières nations, d'autre part. L'analyse de ces pratiques innovantes révèle que, en mettant en place un modèle pédagogique au sein duquel s'articulent quatre éléments particuliers - a) la mise en place d'une architecture particulière, b) le développement de services aux étudiants autochtones, c) l'adoption d'une approche qui lie les dispositifs de formation continue aux programmes universitaires et d) une modularisation des cours-, il a été possible pour l'ENAP d'élaborer des programmes de formation universitaire respectueux des besoins et des réalités autochtones, favorisant, par la même, la réussite des parcours de formation.

\section{INDEX}

Mots-clés : administration publique, autochtones, autonomie gouvernementale, formation continue

\section{AUTEUR}

\section{JEAN-FRANÇOIS SAVARD}

École nationale d'administration publique

Pavillon Alexandre-Taché

283, boulevard Alexandre-Taché, case postale 1250,

Succursale Hull, Gatineau (Québec) J8X 3X7

Jean-Francois.Savard@enap.ca 